\title{
Molecular detection and sequencing of SHV gene encoding for extended-spectrum $\beta$-lactamases produced by multidrug resistance some of the Gram-negative bacteria
}

\author{
Eman A. Khalaf ${ }^{1}$, Mushtak T. S. Al-Ouqaili ${ }^{2}$
}

${ }^{1}$ Departement of Biology, University Of Anbar, College of Education for Pure Sciences, Iraq, ${ }^{2}$ Department of Microbiology, University Of Anbar, College of Medicine, Iraq

Abstract

Background and Objective: The increase in extended-spectrum $\beta$-lactamases (ESBLs) producing microbes in recent years has led to the great challenge for the clinician in the treatment. The study aims to investigate the molecular basis of ESBL encoding-resistant gene, SHV to modern $\beta$-lactams. Also, to detect gene sequencing and to compare their genetic relatedness utilizing phylogenetic analysis. Patients and Methods: A total of 100 clinical isolates are submitting after identification to phenotypic confirmatory double disk synergy technique. Polymerase chain reaction was performed for the determination of SHV gene, and sequencing analysis for the amplified gene is also achieved. Results: A total of 27 isolates, 3 (11.1\%), 15 (55.55\%), and 9 (33.0\%) of Escherichia coli, Klebsiella pneumoniae, and Pseudomonas aeruginosa were positive for a phenotypic and confirmatory test for ESBLs, respectively. SHV gene was detected only in 5 (31.25\%) isolates of P. aeruginosa, 2 (12.5\%) E. coli, and $9(56.25 \%)$ in K. pneumoniae. Conclusions: SHV gene plays an essential role in the resistance of ESBL producer isolates to new $\beta$-lactams. The sequencing of this gene revealed $98-99 \%$ compatibility range with the global standard gene in National Center of Biotechnology Information.

Key words: Extended-spectrum beta-lactamases, gram-negative bacteria, SHV gene

\section{INTRODUCTION}

$\mathrm{I}$ is well documented that the resistance of pathogenic organisms has become a serious problem with failures in the treatment of infectious diseases. ${ }^{[1]}$ The clinically most important $\beta$-lactam resistance mechanism in Gram-negative pathogens is enzymatic inactivation of the antibiotics by $\beta$-lactamases ${ }^{[2]}$ Extended-spectrum $\beta$-lactamase (ESBL) is a class of beta-lactamase enzymes gives widespread resistance to $\beta$-lactam antibiotics including penicillin, cephalosporins, and monobactam, so it compromises the efficacy of all $\beta$-lactams but not to carbapenem. ESBL group of enzymes is found widely and causes a severe infection on human health leading to various diseases. ${ }^{[3]}$ These enzymes stimulate hydrolysis of the $\beta$-lactam ring and thereby inhibit these antibiotics. ${ }^{[4]}$ Among ESBLs, the most widespread and clinically relevant are the class ESBLs of transmission electron microscopy (TEM), SHV, and CTX-M types. TEM and SHV type ESBLs are derived from penicillinases TEM1, TEM-2, and SHV-1 and are characterized by several single amino acid substitutions. ${ }^{[5]}$ ESBLs are frequently plasmid encoded. Plasmids responsible for ESBL production frequently carry genes encoding resistance to other drug classes (for example, aminoglycosides). Therefore, antibiotic options in the treatment of ESBL-producing organisms are extremely limited. ${ }^{[6]}$ Beta-lactamase-producing bacteria can play an important role in polymicrobial infections. They have a direct pathogenic impact in causing the infection as

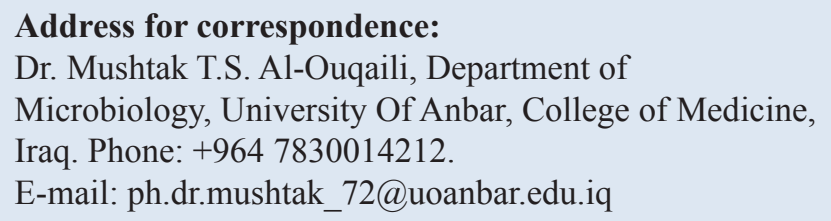

Received: $12-12-2018$

Revised: 24-12-2018

Accepted: 28-12-2018 
well as an indirect effect through their ability to produce the enzyme beta-lactamase. ${ }^{[7]}$ Urinary tract infections, otitis media, abdominal infections, and meningitis are the common and serious diseases caused by these pathogens. ${ }^{[8]}$ Express detection of these resistant organisms offers one of the best solutions to improve patient investigation and to monitor hospital-acquired infection as well as reduce the misuse of antibiotics. ${ }^{[9]}$ The present study aimed to characterize an ESBL-producing isolated from a Gram-negative bacteria using primary screening test for investigation on ESBLs, phenotypic confirmatory analysis for the determination of potentiality of above bacteria to produce ESBL and to determine the existence of SHV gene encoding ESBL by detection of gene expression by polymerase chain reaction (PCR) and to detect the SHV gene sequences of K. pneumoniae, Pseudomonas aeruginosa, and Escherichia coli and to compare their genetic relatedness utilizing phylogenetic analysis

\section{PATIENTS AND METHODS}

The total number of 100 from different clinical sites including burns, wounds, otitis media, and urinary tract infection (UTI) was used for this study and taken information about patients (sex, age, and duration of stay in the hospital, disease, and kind of therapy). These samples are obtaining from AL-Ramadi Teaching Hospital and AL-Karkh Hospital in Baghdad, during a period extending from November 2017 to February 2018. The study proposal was submitted successfully into Ethical Approval Committee in the University of Anbar and obtained the Committee Authentication. The study isolates were bacteriologically diagnosed depending on the conventional, macroscopical, and microscopical in addition to biochemical and confirmatory tests. Identified isolates were stored in a medium containing brain heart infusion broth $20 \%$ glycerol. P. aeruginosa ATCC 27853 and E. coli ATCC 25922 were used as international standard isolates.

\section{Detection of ESBLs}

\section{Primary ESBL screening}

Antibiotic susceptibility tests of all ESBL isolates were performed using a disk diffusion method on Muller-Hinton agar with antibiotic disk of ceftriaxone $(30 \mu \mathrm{g})$, ceftazidime $(30 \mu \mathrm{g})$, imipenem $(10 \mu \mathrm{g})$, meropenem $(10 \mu \mathrm{g})$, cefoxitin $(30 \mu \mathrm{g})$, and aztreonam $(10 \mu \mathrm{g})$. The inoculum to be used in this test was prepared by adding growth from five isolated colonies grown on MacConkey agar plates to $5 \mathrm{ml}$ of nutrient broth, this culture was then incubated for $3 \mathrm{~h}$ to produce a bacterial suspension of moderate turbidity and taken from the growth $10 \mu \mathrm{l}$ by micropipette and added to $10 \mathrm{ml}$ of normal saline that compared with turbidity of readymade $0.5 \mathrm{McF}$ arland tube standard. A sterile swab was used to obtain an inoculum from the standardized culture; this inoculum was then swabbed on Muller-Hinton agar plate.
The antibiotic discs were placed on the surface of the medium evenly with sterile forceps and then incubated at $37^{\circ} \mathrm{C}$ for a full $18 \mathrm{~h}$; then, the inhibition zones were measured and interpreted according to Clinical and Laboratory Standards Institute (CLSI). ${ }^{[10]}$

\section{Confirmatory test for ESBLs}

In the confirmatory test for ESBLs, all isolates which show resistance to third-generation cephalosporins (ceftazidime and ceftriaxone) were submitted to double disc synergy test. In this test, amoxiclav (amoxicillin+ clavulanic acid, $30 / 10 \mu \mathrm{g}$ ) disc was placed in the center of the inoculated plate and ceftazidime $(30 \mu \mathrm{g})$ and cefotaxime $(30 \mu \mathrm{g})$ disks alone are set at $10 \mathrm{~mm}$ distance from the amoxiclav disc, the plates were overnight incubated at $37^{\circ} \mathrm{C}$ for $16-18 \mathrm{~h}$. The isolates interpreted as ESBL if there clear synergy of the edge of the inhibition zone of the antibiotic disc toward the amoxiclav $\operatorname{disc}^{[10]}$ [Figures 1 and 2].

\section{DNA extraction}

SaMag bacterial DNA extraction kit is used with SaMag-12 automatic nucleic acid extraction system for the extraction of genomic DNA from study isolates (Samaga, Cepheid, Italy). The extraction process consisted of steps of lysis, binding, washing, and elution. First, a bacterial suspension was done by taking five colonies from bacteria growth on the MacConkey agar and added to $2 \mathrm{ml}$ brain

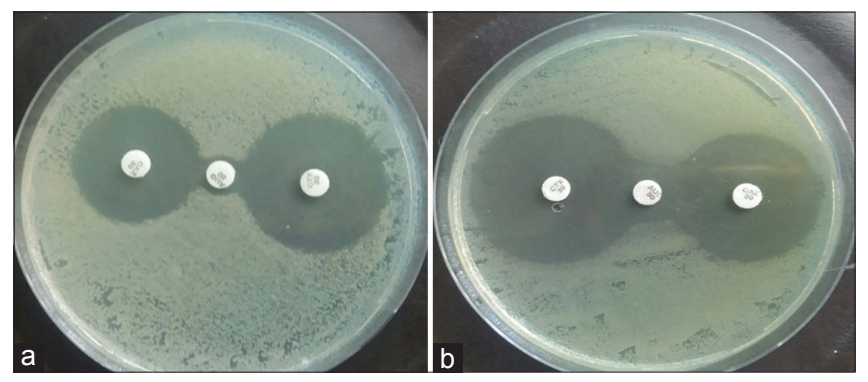

Figure 1: $(a$ and $b)$ The positive result for double disk synergy test between the AUG and CTX, CAZ among clinical study isolates. AUG - (amoxicillin + clavulanic acid, 30/10 $\mu \mathrm{g}$ ), CTX - Cefotaxime $(30 \mu \mathrm{g})$, CAZ - Ceftazidime $(30 \mu \mathrm{g})$

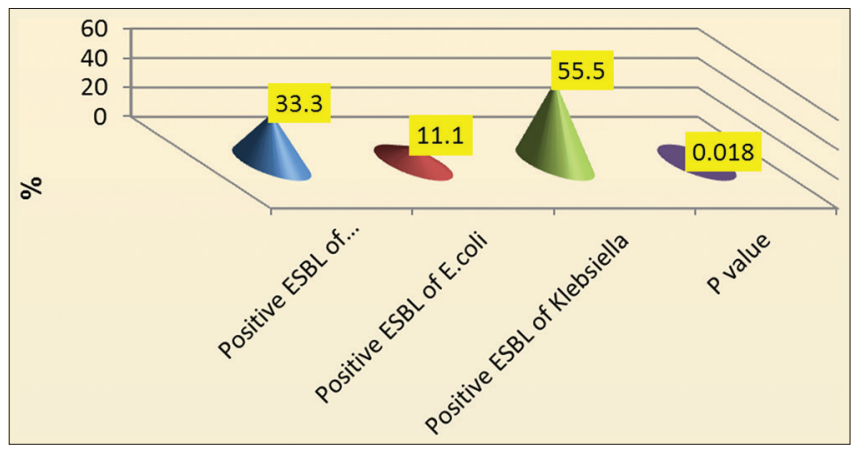

Figure 2: Phenotypic confirmatory test for the production of extended-spectrum $\beta$-lactamases among study isolates 
heart infusion broth and incubated for $24 \mathrm{~h}$ at $37^{\circ} \mathrm{C}$, $1 \mathrm{ml}$ from bacterial suspension was transferred to $1.5 \mathrm{ml}$ microcentrifuge tube at $\times 5000 \mathrm{~g}$ for $5 \mathrm{~min}$, then discard supernatant and added $220 \mu 1$ buffer BL2 to pellet and mix by vortexing for 5-10 s. After that, $200 \mu \mathrm{l}$ suspensions had been taken to sample tube (supplied in the kit) and added $10 \mu \mathrm{l}$ from control positive to all tubes of the device, finally insert these samples tube to automated extraction DNA device (Samag-12).

\section{Molecular method for the detection of ESBLs}

The genomic DNA was used as templates in the specific PCR amplification for the detection of the blaSHV, blaSHV-F (5'-ATTCAGTTCCGTTTCCCAGCGG -3') (GC 54.55\%), (TM $\left.68^{\circ} \mathrm{C}\right)$, and blaSHV-R (5'-AAGATCCACTAT CGCCAGCAG-3') (GC $52.38 \%$ ), (TM 64 C) having product size $231 \mathrm{bp}$. These forward and reverse primers were purchased from $\alpha$-Canada Co., Italy. PCR is performing according to the Maxime PCR Pre-Mix kit, i-Taq DNA Polymerase $5 \mathrm{U} / \mu \mathrm{l}$, dNTPs $2.5 \mathrm{mM}$, reaction buffer $(\times 10)$, and gel loading buffer $\times 10$. About $5 \mu \mathrm{l}$ from Taq PCR PreMix, 10 picomols $/ \mu l(1 \mu \mathrm{l})$ from forwarding primer, 10 picomols $/ \mu \mathrm{l}(1 \mu \mathrm{l})$ from the reverse primer, $1.5 \mu \mathrm{l}$ DNA, $16.5 \mu \mathrm{l}$ distill water to reach final volume $25 \mu \mathrm{l}$ then mixed well by vortex. All tubes transferred into the thermal cycler.

The optimum condition for the detection SHV gene as follows: Initial denaturation at $95^{\circ} \mathrm{C}$ for $3 \mathrm{~min}, 35$ cycles of denaturation at $95^{\circ} \mathrm{C}$ for $45 \mathrm{~s}$, annealing at $50^{\circ} \mathrm{C}$ for $45 \mathrm{~s}$, amplification at $72^{\circ} \mathrm{C}$ for $45 \mathrm{~s}$, and the final extension at $72^{\circ} \mathrm{C}$ for $7 \mathrm{~min}$. The amplified products were separated in 1\% agarose gel and the electrophoresis was performed according to standard procedure.

\section{Sequencing of DNA}

Sequencing of PCR product was achieved by NICEM Company (South Korea) at which the PCR DNA products had been sending with their specific primers in a freezer bag. The sequencing part of this research was designed between the sequence of standard gene BLAST program which is already present at online National Center of Biotechnology Information (NCBI) at http://www.ncbi.nlm.nih.gov and using BioEdit program. The evolutionary analysis was conducted using Molecular Evolutionary Genetics Analysis software version 6 software. ${ }^{[11]}$

\section{Statistical analysis}

Statistical significance is considering with the threshold $P<0.05$. The significant differences were detected using Chi-square; Chi-square test was used to study the association (dependence) between antibiotic susceptibility (sensitivity, resistance, and intermediate), production of ESBL, and prevalence of these types of the genes blaSHV among bacterial isolates.

\section{RESULTS}

A total of 100 clinical samples were collected during this study, out of these specimens, $65(65 \%)$ were positive for culture distributed as follows: $28(43.07 \%)$ $P$. aeruginosa isolates clinical specimens distributed as follows: $2(7.1 \%)$ isolates from wounds, $9(32.1 \%)$ from ear, and $17(60.7 \%)$ from burn, and $25(38.4 \%)$ isolates of Klebsiella distributed as follows: $19(76 \%)$ isolates from burn, $5(20 \%)$ isolates from UTI, and $1(4 \%)$ isolates from ear, and $12(18.4 \%)$ isolates from $E$. coli distributed as follows: $5(41.6 \%)$ isolates from burn and $7(58.3 \%)$ isolates from UTI, and others isolates from UTI including 12 isolates were Gram-positive bacteria, and 23 isolates were without growth (culture negative). Negative growth is due to the fact that patients are antimicrobials chemotherapy during culture time. These isolates are screening using the Kirby-Bauer disk diffusion method by measuring the diameter of inhibition zones around antibiotic discs showed that $K$. pneumoniae were resistance to ceftriaxone $22(88 \%)$, ceftazidime $21(84 \%)$, aztreonam $22(88 \%)$, cefepime $12(92.3)$, and more sensitive to meropenem and imipenem. E. coli were resistance to ceftriaxone $12(100 \%)$, ceftazidime $10(83.3 \%)$, aztreonam $12(100 \%)$, and cefoxitin $10(83.3 \%)$ and show resistance to meropenem $2(16.7 \%)$ and $3(25 \%)$ for imipenem. Pseudomonas showed resistance to ceftriaxone 20 (71.4\%), ceftazidime $19(67.9 \%)$, aztreonam $6(21.4 \%)$, cefepime $13(68.4 \%)$, and cefoxitin $(100 \%)$ and one isolate shows resistance to imipenem $1(3.6 \%)$. In the confirmatory test, 27 of $60(45 \%)$ are identified as ESBL positive; $9(33.3 \%)$ of P. aeruginosa 15 (55.5\%) of Klebsiella and 3 (11.1\%) of $E$. coli. Statistical analysis shows a statistically significant $(P>0.05)(0.018)$.

In the genetic part, PCR assay has been used to detect the presence of extended- $\beta$-lactamases gene blaSHV in $K$. pneumoniae, E. coli, and P. aeruginosa isolate from clinical environment. In the present study, 16 of 27 isolates from K. pneumoniae, E. coli, and Pseudomonas have carried SHV gene as follows: $9(56.25 \%)$ in K. pneumoniae, $5(31.25 \%)$ in $P$. aeruginosa, and $2(12.5 \%)$ in E. coli as represented in [Figures 3 - 5].

\section{Results of sequencing and phylogenetic analysis of SHV gene}

The study result revealed of six isolates no 1, 13, 23, 9, 3, and 10 of amplified SHV gene, DNA which was sent for sequencing and phylogenetic. Alignment study of SHV gene which encoded for $K$. pneumoniae isolates (No. 1, 13, and 23) revealed that there is closely related genotype to the novel ones which isolated from Senegal, Spain: Madrid, Australia, China, Iran, France, Brazil, India, Thailand: Chonburi, USA: Chicago, IL, Germany: Hamburg, and Vietnam: Hanoi deposit in the Gene Bank with accession numbers documented in the following Table 1. Further, the 
sequencing of these genes appeared $98 \%$ compatibility range in comparison with the global standard gene in a gene bank in NCBI. On the other hand, SHV gene which

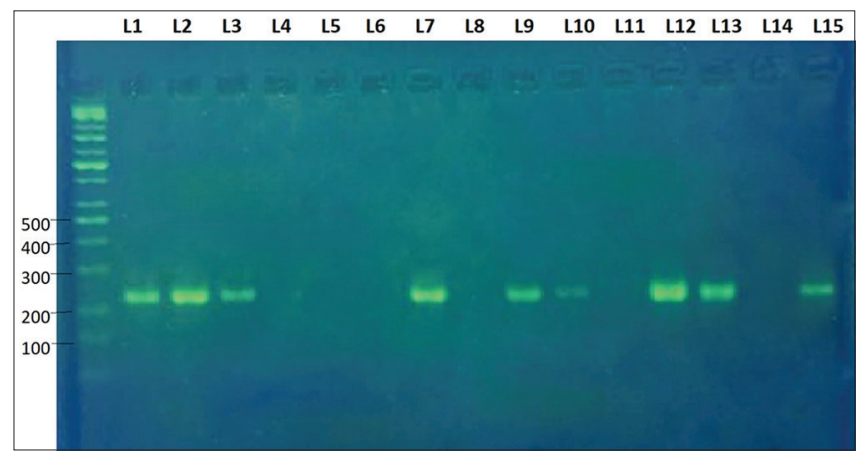

Figure 3: Distribution of SHV gene in study isolates. Agarose gel electrophoresis (2\%) with Novel Juice dye agarose at 5 volt $/ \mathrm{cm}^{2}$. $\times 1$ tris/borate/ethylenediaminetetraacetic acid buffer for 1:30 h. Bands with SHV gene (231 bp) obtained from $K$. pneumoniae isolates, which revealed positive results are represented by $L 1, L 2, L 3, L 7, L 9, L 10, L 12, L 13$, and L15 while L5, L6, L8, L11, and L14 were negative results. DNA ladder with 100-2000 bp on the left and right used as a molecular weight marker for DNA

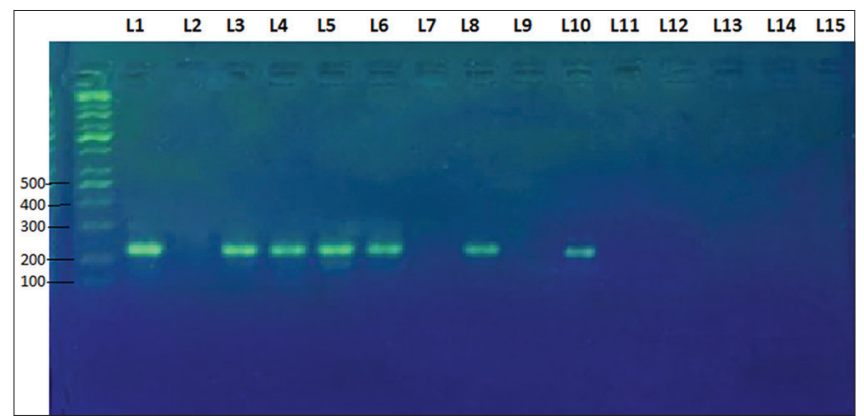

Figure 4: Distribution of SHV gene in study isolates. Agarose gel electrophoresis (2\%) with Novel Juice dye agarose at 5 volt $/ \mathrm{cm}^{2} . \times 1$ tris/borate/ethylenediaminetetraacetic acid buffer for $1: 30 \mathrm{~h}$. Bands with SHV gene (231 bp) obtained from $P$. aeruginosa and $E$. coli. Isolates, which revealed that positive results are represented by L1, L3-L6, L8, and L10 while L5, L6, L8, L11, and L14 were negative results. DNA ladder with 100-2000 bp on the left and right used as a DNA molecular weight marker

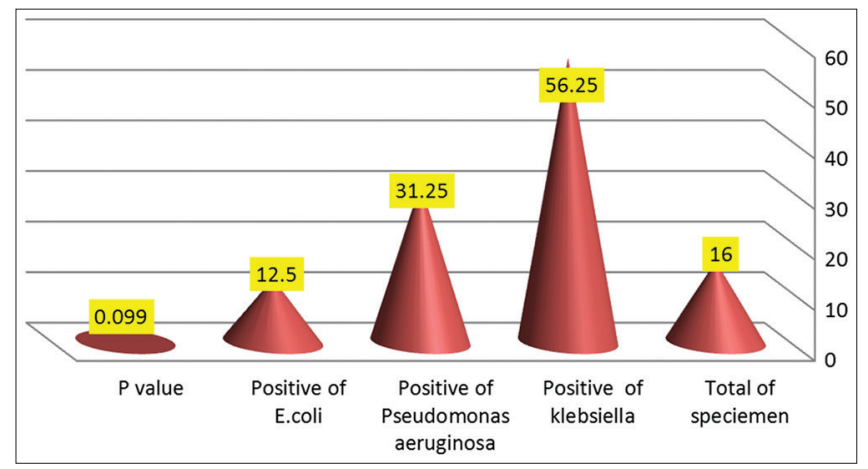

Figure 5: Distribution of the positive cases of blaSHV among study isolates encoded for $P$. aeruginosa isolated No. 9 and 3 revealed that there are similar with those countries with compatibility range (99\%) in Brazil, Egypt, United Arab Emirates, India, Japan, Tunisia, France, and Switzerland, followed by Greece (98\%), the USA (74\%), Brazil: Belo Horizonte (74\%), and Colombia (71\%) with accession numbers documented in the following Table 2. Furthermore, SHV gene which encoded for $E$. coli isolates No. 10 revealed that there is closely related genotype to the novel ones which isolated from India, Iran, China, France, Australia, the Netherlands, and

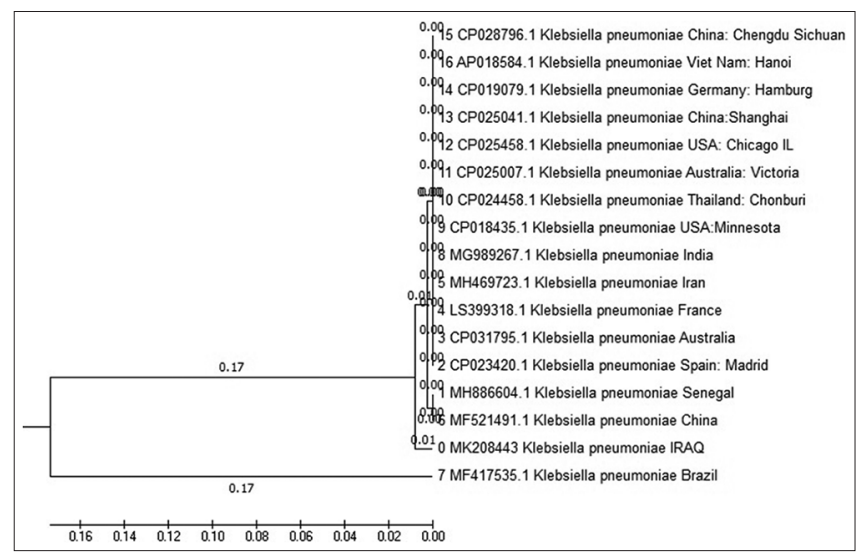

Figure 6: Phylogenetic analysis tree of Iraqi SHV genes which encoded for Klebsiella pneumoniae genetic distance with other global

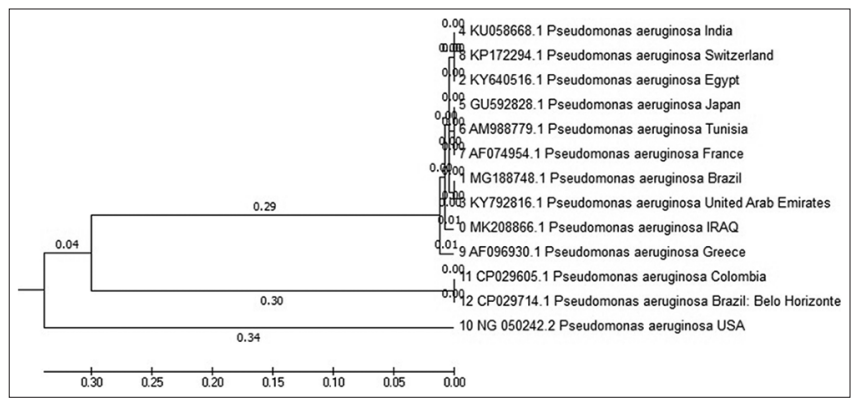

Figure 7: Phylogenetic analysis tree of Iraqi SHV genes which encoded for Pseudomonas aeruginosa genetic distance with other global

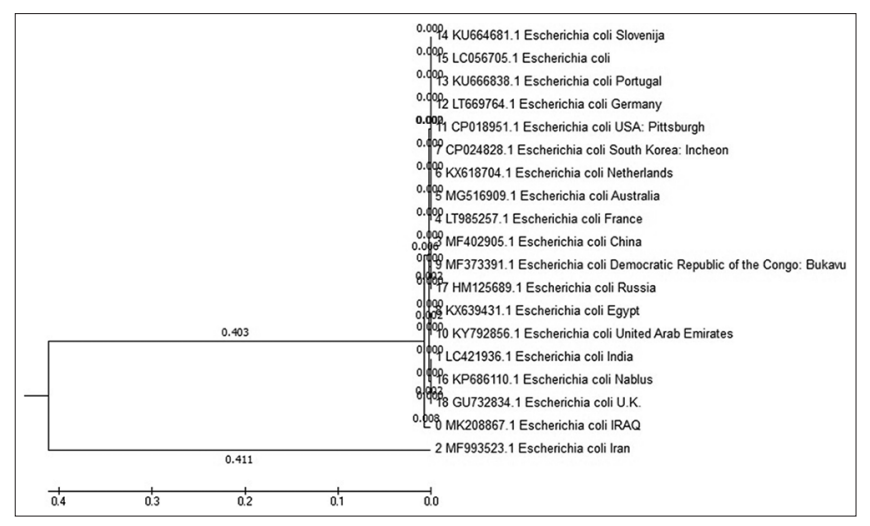

Figure 8: Phylogenetic analysis tree of Iraqi SHV genes which encoded for Escherichia coli genetic distance with other global 
Table 1: The BLAST results of SHV gene $K$. pneumoniae in the gene bank, and compatibility of DNA sequences obtained from NCBI

\begin{tabular}{lllllc} 
S. No & Accession & Gene & Country & Source & Compatibility (\%) \\
\hline 1 & ID: MH886604.1 & (blaSHV) gene & Senegal & K. pneumoniae & 98 \\
2 & ID: CP023420.1 & (blaSHV) gene & Spain: Madrid & K. pneumoniae & 98 \\
3 & ID: CP031795.1 & (blaSHV) gene & Australia & K. pneumoniae & 98 \\
4 & ID: LS399318.1 & (blaSHV) gene & France & K. pneumoniae & 98 \\
5 & ID: MH469723.1 & (blaSHV) gene & Iran & K. pneumoniae & 98 \\
6 & ID: MF521491.1 & (blaSHV) gene & China & K. pneumoniae & 98 \\
7 & ID: MG653189.1 & (blaSHV) gene & Brazil & K. pneumoniae & 98 \\
8 & ID: MG989267.1 & (blaSHV) gene & India & K. pneumoniae & 98 \\
9 & ID: CP018435.1 & (blaSHV) gene & USA: Minnesota & K. pneumoniae & 98 \\
10 & ID: CP024458.1 & (blaSHV) gene & Thailand: Chonburi & K. pneumoniae & 98 \\
11 & ID: CP025007.1 & (blaSHV) gene & Australia: Victoria & K. pneumoniae & 98 \\
12 & ID: CP025041.1 & (blaSHV) gene & USA: Chicago, IL & K. pneumoniae & 98 \\
13 & ID: CP025458.1 & (blaSHV) gene & China: Shanghai & K. pneumoniae & 98 \\
14 & ID: CP019079.1 & (blaSHV) gene & Germany: Hamburg & K. pneumoniae & 98 \\
15 & ID: CP028796.1 & (blaSHV) gene & China: Chengdu, Sichuan & K. pneumoniae & 98 \\
16 & ID: AP018584.1 & (blaSHV) gene & Vietnam: Hanoi & K. pneumoniae & 98
\end{tabular}

NCBI: National Center Biotechnology Information, K. pneumoniae: Klebsiella pneumoniae

Table 2: The BLAST results of SHV gene which encoded for $P$. aeruginosa in the gene bank, and compatibility of DNA sequences obtained from NCBI

\begin{tabular}{lllllc} 
S. No & Accession & Gene & Country & Source & Compatibility (\%) \\
\hline 17 & ID: MG188748.1 & (blaSHV) gene & Brazil & P. aeruginosa & 99 \\
18 & ID: KY640516.1 & (blaSHV) gene & Egypt & P. aeruginosa & 99 \\
19 & ID: KY792816.1 & (blaSHV) gene & United Arab Emirates & P. aeruginosa & 99 \\
20 & ID: KU058668.1 & (blaSHV) gene & India & P. aeruginosa & 99 \\
21 & ID: GU592828.1 & (blaSHV) gene & Japan & P. aeruginosa & 99 \\
22 & ID: AM988779.1 & (blaSHV) gene & Tunisia & P. aeruginosa & 99 \\
23 & ID: AF074954.1 & (blaSHV) gene & France & P. aeruginosa & 99 \\
24 & ID: KP172294.1 & (blaSHV) gene & Switzerland & P. aeruginosa & 99 \\
25 & ID: AF096930.1 & (blaSHV) gene & Greece & P. aeruginosa & 98 \\
26 & ID: NG_050242.2 & (blaSHV) gene & USA & P. aeruginosa & 74 \\
27 & ID: CP029605.1 & (blaSHV) gene & Colombia & P. aeruginosa & 71 \\
28 & ID: CP029714.1 & (blaSHV) gene & Brazil: Belo Horizonte & P. aeruginosa & 74 \\
\hline
\end{tabular}

NCBI: National Center Biotechnology Information, P. aeruginosa: Pseudomonas aeruginosa

South Korea: Incheon. Egypt, Democratic Republic of the Congo: Bukavu, United Arab Emirates, USA: Pittsburgh, Germany, Portugal, Slovenija, Japan, Nablus, Russia, and the U.K. deposit in the Gene Bank with accession numbers documented in the following Table 3. The sequencing of these genes appeared $99 \%$ compatibility range in comparison with the global standard gene in a gene bank in NCBI. Furthermore, the sequencing and BLAST analysis of partial SHV gene and types of polymorphism for the gene are reflecting in Table 4 [Figures 6-8].

\section{DISCUSSION}

It is well known that Gram-negative bacilli are the most commonly isolated living organisms from clinical specimens, and due to the high production of ESBL in this group, this requires laboratory testing methods to correctly detect these enzymes for good management. ${ }^{[12]}$ In our study, GNBs distribution was as follows: E. coli were $12(18.4 \%)$ and K. pneumoniae was 25 (38.4\%). In our research, P. aeruginosa accounted for the most ESBL-producing organisms in 
Table 3: The BLAST results of SHV gene which encoded for E. coli in the gene bank, and compatibility of DNA sequences obtained from $\mathrm{NCBI}$

\begin{tabular}{lllllc} 
S. No & Accession & Gene & Country & Source & Compatibility (\%) \\
\hline 29 & ID: LC421936.1 & (blaSHV) gene & India & E. coli & 99 \\
30 & ID: MF993523.1 & (blaSHV) gene & Iran & E. coli & 99 \\
31 & ID: MF402905.1 & (blaSHV) gene & China & E. coli & 99 \\
32 & ID: LT985257.1 & (blaSHV) gene & France & E. coli & 99 \\
33 & ID: MG516909.1 & (blaSHV) gene & Australia & E. coli & 99 \\
34 & ID: KX618704.1 & (blaSHV) gene & Netherlands & E. coli & 99 \\
35 & ID: CP024828.1 & (blaSHV) gene & South Korea: Incheon & E. coli & 99 \\
36 & ID: KX639431.1 & (blaSHV) gene & Egypt & E. coli & 99 \\
37 & ID: MF373391.1 & (blaSHV) gene & Democratic Republic of the Congo: Bukavu & E. coli & 99 \\
38 & ID: KY792856.1 & (blaSHV) gene & United Arab Emirates & E. coli & 99 \\
39 & ID: CP018951.1 & (blaSHV) gene & USA: Pittsburgh & E. coli & 99 \\
40 & ID: LT669764.1 & (blaSHV) gene & Germany & E. coli & 99 \\
41 & ID: KU666838.1 & (blaSHV) gene & Portugal & E. coli & 99 \\
42 & ID: KU664681.1 & (blaSHV) gene & Slovenija & E. coli & 99 \\
43 & ID: LC056705.1 & (blaSHV) gene & Japan & E. coli & 99 \\
44 & ID: KP686110.1 & (blaSHV) gene & Nablus & E. coli & 99 \\
45 & ID: HM125689.1 & (blaSHV) gene & Russia & E. coli & 99 \\
46 & ID: GU732834.1 & (blaSHV) gene & U.K. & E. coli & 99 \\
\hline
\end{tabular}

NCBI: National Center Biotechnology Information, E. coli: Escherichia coli

Table 4: Type of polymorphism of SHV genes which encoded for three study isolates of $K$. pneumoniae, two isolates of $P$. aeruginosa, and one isolate of $E$. coli

\begin{tabular}{|c|c|c|c|c|c|}
\hline Number and source of isolates & Type of substitution & Location & Identities (\%) & Nucleotide & Sequence ID \\
\hline \multirow[t]{2}{*}{1 (K. pneumoniae) } & Transition & 272 & 99 & $C>T$ & ID: MH469723.1 \\
\hline & Transversion & 440 & - & $\mathrm{T}>\mathrm{A}$ & \\
\hline \multirow[t]{3}{*}{13 (K. pneumoniae) } & Transition & 272 & 98 & $C>T$ & ID: MH469723.1 \\
\hline & Transition & 294 & - & $\mathrm{G}>\mathrm{A}$ & \\
\hline & Transversion & 440 & - & $\mathrm{T}>\mathrm{A}$ & \\
\hline 23 (K. pneumoniae) & Transition & 272 & 99 & $C>T$ & ID: MH469723.1 \\
\hline \multirow[t]{2}{*}{9 (P. aeruginosa) } & Transversion & 335 & 99 & $\mathrm{~T}>\mathrm{A}$ & ID: MG188748.1 \\
\hline & Transition & 354 & - & $A>G$ & \\
\hline 3 (P. aeruginosa) & Transversion & 334 & 99 & $C>G$ & ID: MG188748.1 \\
\hline \multirow[t]{3}{*}{$10($ E. coli) } & Transition & 539 & 99 & $A>G$ & ID: MF993523.1 \\
\hline & Transversion & 505 & - & $\mathrm{T}>\mathrm{A}$ & \\
\hline & Transversion & 465 & - & $A>T$ & \\
\hline
\end{tabular}

Klebsiella pneumoniae: K. pneumonia, Pseudomonas aeruginosa: $P$. aeruginosa

all clinical samples 28 (43.07\%). After screening all the GNBs, according to CLSI recommended screening criteria, $60(92.3 \%)$ were found to be potential ESBL producers. However, ESBL production was confirmed in only 27 (45\%) by the phenotypic confirmatory method. Thus, in our study, the confirmatory method could not detect ESBL production in 33 isolates (55\%). This results may be due to additional resistance mechanisms may be due to the production of other types of enzymes such as ambler Class $C \beta$-lactamases as concluded in a study by Al-Ouqaili et al., ${ }^{[13]}$ and MBL enzymes in a study laid down by Al-Ouqaili et al. ${ }^{[14]}$ or by another mechanism for resistance such as biofilm in a survey by Al-Ouqaili et al..$^{[15]}$

Nine $(33.3 \%)$ of $P$. aeruginosa isolates were ESBL positive, which is in agreement with those observed by Hakemi et al., ${ }^{[16]}$ which showed that ESBL positive were $31.1 \%$. However, it is in contrast with two other studies in Iran that 
their results were lower (18\%) and higher (42.8\%) than our result this discrepancy may be related to more usage of betalactam drugs and the time of the study. ${ }^{[17,18]}$ In our study, it was observed that $P$. aeruginosa was resistant to ceftazidime and cefepime at $67.9 \%$ and $68.4 \%$ respectively. This result was in agreement with those observed by Hakemi and associates ${ }^{[16]}$ with the percentage of resistance $66 \%$ and $67 \%$ for both of ceftazidime and cefepime respectively. Nithyalakshmi et al. ${ }^{[19]}$ agreed with our study in finding low resistance to imipenem (3.4\%). The resistance tool of problematic infections affiliated from $P$. aeruginosa to imipenem and meropenem may be due to diminished expression of an OprD-like protein which works as a carbapenem-specific channel and expressed by the OprD gene. This interpretation is reflected clearly in a study laid down by Al-Ouqaili et al. ${ }^{[20]}$

$K$. pneumoniae isolates were resistant to ceftriaxone $22(88 \%)$ and ceftazidime $21(84 \%)$, these results were a similarity with Natoubi et al..$^{[21]}$ showed that the resistance of $K$. pneumoniae to ceftriaxone and ceftazidime was $81.08 \%$ and $81.08 \%$, respectively. In this study, $K$. pneumoniae was the most frequent ESBL producer, Klebsiella ESBL producer was $55.5 \%$ that similarity with Al-gamy et al. ${ }^{[22]}$ showed that ESBL production was $55 \%$ isolates of $K$. pneumoniae isolated from hospital-acquired infections in Riyadh. However, these contrast with Saha et al.,. ${ }^{[23]}$ ESBL producers were observed among Klebsiella spp. (75\%) and with AL-Subol and Youssef, ${ }^{[24]}$ they were detected $(67.5 \%)$ of $K$. pneumoniae, different rates for the prevalence of ESBL-producing $K$. pneumoniae are detecting in the variation difficult to explain but may be due to differences in antibiotic exhaustion and a variety of specimens handling time. Further, E. coli isolates was resistant to the antimicrobial agents meropenem, ceftazidime, cefoxitin, ceftriaxone, aztreonam, and cefoxitin with percentage of resistance $16.7 \%, 83.3,83.3 \%, 100 \%$, and 100, respectively. This result was in agreement with those observed by Hassan et al.. ${ }^{[25]}$ who reported that the resistance rates to ceftazidime, ceftriaxone, and aztreonam among E. coli isolates were $97.8 \%, 98.6 \%$, and $98.5 \%$ respectively. In another study laid down by Devrim et al.. ${ }^{[26]}$, the researchers documented that the rate of resistance of meropenem and imipenem was $7.4 \%$.

In the present study, $11.1 \%$ isolates of $E$. coli were ESBL producers agree with the investigation by Batchoun et al..$^{[27]}$ observed that ESBL production of E. coli was $10.8 \%$. In compare to our study, the study by Devrim and associates ${ }^{[26]}$ reported that ESBL present in 27 (72.9\%) of study isolates. Also, in a study of Chourasia and co-workers ${ }^{[28]}$ documented that the prevalence rate of ESBL in E. coli was (60.4\%), while Balan ${ }^{[29]}$ observed that $19.6 \%$ isolates of $E$. coli were ESBL producers.

In the genetic part of this study, $16(59.25 \%)$ of 27 isolates are carrying carried SHV gene and $11(40.7 \%)$ isolates expressed a negative result to the genes used in this study due to other genes encoding ESBLs not used in this study. The results of the blaSHV gene among Klebsiella isolates were $56,25 \%$ this agree with Haji et al..$^{[30]}$ and $55.5 \%$ of $K$. pneumoniae isolates. These results were different from another study by Hassan et al. ${ }^{[25]}$ showed that SHV gene was $86 \%$ of $K$. pneumoniae isolates. The rate of SHV in this study was a little higher than that reported in a Turkish hospital Dagi et al. ${ }^{[31]}$ showed that SHV in K. pneumoniae isolates were $24.4 \%$. SHV gene has appeared in E. coli with the low ratio (12.5\%). This ratio was in agreement with those observed by study in Iran Moosavian and Deiham ${ }^{[32]}$ SHV was $9 \%$. These results were different from other studies Hassan et al., ${ }^{[25]}$ detect the prevalence of SHV in E. coli was $22 \%$, and study by Mirzaee et al. ${ }^{[33]}$ showed that the prevalence of SHV was $31.6 \%$.

The study revealed that the prevalence rate of Pseudomonas aeruginosa was $(31.25 \%)$. This result was similar to those observed by Toupkanlou, et al., ${ }^{[34]}$ who reported the prevalence rate is $36 \%$. In contrast to our study, a study by Ghaima and Abdulhassan ${ }^{[35]}$ detection the prevalence of ESBL-SHV gene in P. aeruginosa was $52.3 \%$, Bahmani and Ramazanzadeh $^{[36]}$ detected that the prevalence of ESBLSHV gene in $P$. aeruginosa was $10.57 \%$ and in a study by Bokaeian et al., ${ }^{[37]}$ blaSHV was $6.6 \%$, and Chika and Agbakoba $^{[38]}$ was $43.5 \%$.

The phylogenetic tree revealed that the SHV encoded for $K$. pneumoniae revealed that the compatibility range (98\%), and SHV encoded for $E$. coli revealed that the compatibility range $(99 \%)$ while SHV gene which encoded for P. aeruginosa isolated showed a variation in the compatibility range with countries (99\%) in Brazil, Egypt, United Arab Emirates, India, Japan, Tunisia, France, and Switzerland, followed by Greece (98\%), the USA (74\%), Brazil: Belo Horizonte (74\%), and Colombia (71\%).

\section{CONCLUSIONS}

SHV encoding resistance is detecting in high prevalence, particularly among $K$. pneumoniae isolates. Furthermore, SHV plays an essential role in the resistance of ESBL producer isolates to new $\beta$-lactams. Further, the sequencing of these genes revealed $98-99 \%$ compatibility range with the global standard gene in gene bank documented in NCBI. The phylogenetic tree of SHV gene which encoded for $K$. pneumoniae, $P$. aeruginosa, and E. coli revealed that the compatibility ranges were (98\%), (71-99\%) and (99\%) for the above bacteria respectively in compare with the global standard gene in gene bank documented in NCBI.

\section{REFERENCES}

1. Alekshun MN, Levy SB. Molecular mechanisms of antibacterial multidrug resistance. Cell 2007;128:1037-50.

2. BushK. Proliferation and significance of clinically relevant 
$\beta$-lactamases. Ann N Y Acad Sci 2013;1277:84-90.

3. Saravanan M, Ramachandran B, Barabadi $H$. The prevalence and drug resistance pattern of extended spectrum $\beta$-lactamases (ESBLs) producing Enterobacteriaceae in Africa. Microb Pathog 2018;114:180-92.

4. Kaviyarasan G, Kcp R, Prasanth D. Prevalence of cephalosporin-resistant gram-negative bacilli from clinical samples. Asian J Pharm Clin Res 2016;9:176-8.

5. Rubtsova MY, Ulyashova MM, Bachmann TT, Schmid RD, Egorov AM. Multiparametric determination of genes and their point mutations for identification of beta-lactamases. Biochemistry (Mosc) 2010;75:1628-49.

6. Akyala AI, Alsam S. Extended spectrum beta-lactamase producing strains of Salmonella species a systematic review. J Microbiol Res 2015;5:57-70.

7. Thenmozhi S, Moorthy K, Sureshkumar BT, Suresh M. Antibiotic resistance mechanism of ESBL producing Enterobacteriaceae in clinical field: A review. Int J Pure Appl Biosci 2014;2:207-26.

8. Martin CA. Gram-negative bacteria. In: Mainous AG, Pomeroy C, editors. Management of Antimicrobials in Infectious Disease. Lexington, USA: Springer Science; 2010. p. 45-59.

9. Gazin M, Paasch F, Goossens H, Malhotra-Kumar S, MOSAR WP2 and SATURN WP1 Study Teams. Current trends in culture-based and molecular detection of extended-spectrum- $\beta$-lactamase-harboring and carbapenem-resistant Enterobacteriaceae. J Clin Microbiol 2012;50:1140-6.

10. Clinical and Laboratory Standards Institute (CLSI). Performance Standards for Antimicrobial Susceptibility Testing. 27 $7^{\text {th }}$ ed. Wayne: CLSI Supplement M100; 2017.

11. Tamura K, Stecher G, Peterson D, Filipski A, Kumar S. MEGA6: Molecular evolutionary genetics analysis version 6.0. Mol Biol Evol 2013;30:2725-9.

12. Siddiqui N, Bhakre J, Damle A, Bajaj J. Prevalence of extended-spectrum beta-lactamase (ESBL) producing gram-negative bacilli from various clinical isolates. IOSR J Dent Med Sci 2014;13:8-11.

13. Al-Ouqaili MT, Al-Kubaisy SH, Al-Ani AJ. Detection of extended spectrum and ambler class $\mathrm{C}$ beta-lactamases among beta-lactam resistant Klebsiella species: Genetic aspects. Egypt Soc Exp Bot 2011;7:299-308.

14. Al-Ouqaili MT, Al-Taei SH, Al-Najjar A. Molecular detection of medically important carbapenemases genes expressed by metallo- $\beta$-lactamase producer isolates of Pseudomonas aeruginosa and Klebsiella pneumoniae. Asian J Pharm 2018;12:S991-1001.

15. Al-Ouqaili MT, Al-Quhli SQ, Al-Izzy MY. The Role of milleri streptococci in the formation of cariogenic biofilm: Bacteriological aspects. Jordan J Biol Sci 2011;4:165-72.

16. Hakemi VM, Hallajzadeh M, Fallah F, Hashemi A, Goudarzi H. Characterization of the extended-spectrum beta-lactamase producers among non-fermenting gramnegative bacteria isolated from burnt patients. Arch Hyg
Sci 2013;2:1-6.

17. Jabalameli F, Mirsalehian A, Sotoudeh N, Jabalameli L, Aligholi M, Khoramian B, et al. Multiple-locus variable number of tandem repeats (VNTR) fingerprinting (MLVF) and antibacterial resistance profiles of extended spectrum beta lactamase (ESBL) producing Pseudomonas aeruginosa among burnt patients in Tehran. Burns 2011;37:1202-7.

18. Vahdani M, Azimi L, Asghari B, Bazmi F, Rastegar Lari A. Phenotypic screening of extended-spectrum B-lactamase and metallo-ß-lactamase in multidrugresistant Pseudomonas aeruginosa from infected burns. Ann Burns Fire Disasters 2012;25:78-81.

19. Nithyalakshmi J, Vidhyarani R, Mohanakrishnan K, SumathiG. ESBL producing Pseudomonas aeruginosa in clinical specimens: Is it a scary nightmare or paper tiger? Indian J Microbiol Res 2016;3:287-91.

20. Al-Ouqaili MT, Jal'oot AS, Badawy AS. Identification of an OprD and bla ${ }_{\mathrm{IMP}}$ gene-mediated carbapenem resistance in Acinetobacter baumannii and Pseudomonas aeruginosa among patients with wound infections in Iraq. Asian J Pharm 2018;12:S959-65.

21. Natoubi S, Barguigua A, Zriouil SB, Baghdad N, Timinouni M, Hilali A, Zerouali K. The incidence of extended-spectrum be-ta-lactamase-producing Klebsiella pneumoniae among patients and in the environment of Hassan II hospital, Settat, Morocco. Adv Microbiol 2016;6:152-61.

22. Al-Agamy MH, Shibl AM, Tawfik AF. Prevalence and molecular characterization of extended-spectrum betalactamase-producing Klebsiella pneumoniae in Riyadh, Saudi Arabia. Ann Saudi Med 2009;29:253-7.

23. Saha MR, Jhora, ST, Khan TM, Paul S, Chowdhury D. Detection of SHV Gene from extended-spectrum betalactamases (ESBLs) producing isolates in a tertiary care hospital. Bangladesh J Med Microbiol 2014;8:14-8.

24. AL-Subol I, Youssef N. Prevalence of CTX-M, TEM and SHV beta-lactamases in clinical isolates of Escherichia Coli and Klebsiella Pneumoniae isolated from Aleppo University Hospitals, Aleppo, Syria. Arch Clin Infect Dis 2015;10:1-6.

25. Hassan MI, Alkharsah KR, Alzahrani AJ, Obeid OE, Khamis AH, Diab A, et al. Detection of extended spectrum beta-lactamases-producing isolates and effect of AmpC overlapping. J Infect Dev Ctries 2013;7:618-29.

26. Devrim F, Serdaroğlu E, Çağlar İ, Oruç Y, Demiray N, Bayram N, et al. The emerging resistance in nosocomial urinary tract infections: From the pediatrics perspective. Mediterr J Hematol Infect Dis 2018;10:e2018055.

27. Batchoun RG, Swedan SF, Shurman AM. Extended spectrum beta-lactamases among gram-negative bacterial isolates from clinical specimens in three major hospitals in Northern Jordan. Int J Microbiol 2009;2009:513874.

28. Chourasia E, Singh KP, Kher SK. Extended spectrum beta $(\beta)$-lactamases in clinical isolates of gram-negative bacilli in Ajman, United Arab Emirates. Gulf Med J 2015;4:14-21. 
29. Balan K. Detection of extended spectrum $\beta$-lactamase among gram-negative clinical isolates from a tertiary care hospital in South India. Int J Res Med Sci 2013;1:28-30.

30. Haji HS, Jalal TS, Omer AS, Mawlood HA. Molecular detection of SHV-Type ESBL in E. coli and $K$. pneumoniae and their antimicrobial resistance profile. Zanco J Med Sci 2018;22:262-72.

31. Dagi HT, Al Dulaimi AA, Kus H, Seyhan T, Findik D, Tuncer I, Arslan U. Genotype distribution of extended Spectrum $\beta$-lactamase producing Escherichia coli and Klebsiella pneumoniae. Biomed Res 2015;26:235-8.

32. Moosavian M, Deiham B. Distribution of TEM, SHV and CTX-M Genes among ESBL-producing Enterobacteriaceae isolates in Iran. Afr J Microbiol Res 2012;6:5433-9.

33. Mirzaee M, Eftekhari R, Taghizadeh N, Mehrabi MR. Relationship between presence of genes encoding ESBLs and antimicrobial susceptibility pattern in Escherichia coli clinical isolates. Iran J Med Microbiol 2016;10:8-15.

34. Toupkanlou SP, Peerayeh SN, Mahabadi RP. Class A and $\mathrm{D}$ extended-spectrum $\beta$-lactamases in imipenem resistant Pseudomonas aeruginosa isolated from burn patients in Iran. Jundishapur J Microbiol 2015;8:e18352.

35. Ghaima KK, Abdulhassan AA, Mahdi ZH. Molecular study of extended-spectrum beta-lactamase (ESBL) genes in Pseudomonas aeruginosa isolate from burns. Biochem Cell Arch 2018;18:721-7.

36. Bahmani N, Ramazanzadeh R. Detection of SHV type extended-spectrum B-lactamase and risk factors in Pseudomonas aeruginosa clinical isolates. Pak J Med Sci 2013;29:788-92.

37. BokaeianM,ZahedaniSS, BajgiranMS, Moghaddam AA. The frequency of PER, VEB, SHV, TEM and CTX-M genes in resistant strains of Pseudomonas aeruginosa producing extended spectrum $\beta$-lactamases. Jundishapur J Microbiol 2015;8:1-6.

38. Chika E, Agbakoba NR. Prevalence and identification of bla VEB-1, bla SHV and bla CTX-m in Pseudomonas aeruginosaIsolate from hospitals in Onitsha metropolis using polymerase chain reaction technique. Glob J Mol Sci 2016;11:1-6.

Source of Support: Nil. Conflict of Interest: None declared. 